\title{
Laboratory analogs of carbonaceous matter: Soot and its precursors and by-products
}

\author{
Cornelia Jäger ${ }^{1}$, Harald Mutschke ${ }^{2}$, Isabel Llamas-Jansa ${ }^{2}$ \\ Thomas Henning $^{3}$, and Friedrich Huisken ${ }^{1}$ \\ ${ }^{1}$ Laboratory Astrophysics Group of the Max Planck Institute for Astronomy \\ at the Institute of Solid State Physics, Friedrich Schiller University Jena \\ Helmholtzweg 3, 07743 Jena, Germany \\ email: Cornelia.Jaeger@uni-jena.de \\ ${ }^{2}$ Astrophysical Institute and University Observatory, FSU Jena \\ Schillergässchen 3, 07745 Jena, Germany \\ ${ }^{3}$ Max Planck Institute for Astronomy \\ Königstuhl 17, D-69117 Heidelberg, Germany
}

\begin{abstract}
Carbonaceous materials have been prepared in the laboratory by laser-induced pyrolysis of a mixture of hydrocarbons under different conditions and laser ablation of graphite in reactive gas atmospheres. We have investigated the soluble and insoluble parts of the condensed carbon powders with several spectroscopic and chromatographic methods in order to obtain information on the composition of the condensate. The results of these experiments have demonstrated that, at temperatures lower than $1700 \mathrm{~K}$, the pyrolysis by-products are mainly PAHs, whereas at higher temperatures fullerenes and polyyne-based compounds are formed. The experimental findings point to different soot formation mechanisms with variable intermediates and end products. It has been found that soot extracts can contain more than 65 different polycyclic aromatic hydrocarbons (PAHs). Eventually, the study of the condensation pathways of soot particles and their precursors and by-products will permit the prediction of the spectral properties of carbonaceous matter in space.
\end{abstract}

Keywords. Laboratory, dust, extinction, infrared, stars

\section{Carbonaceous cosmic dust analogs}

Plenty of different analogs for the carbonaceous cosmic dust have been produced and characterized in the laboratories. Their spectral properties have been measured and compared to observations in order to obtain information on the exact composition and processing of the cosmic dust in different astrophysical environments. Hydrogenated amorphous carbon (HAC) was first introduced as a possible cosmic dust analog by Duley \& Williams (1981). Sakata et al. (1983) produced a carbonaceous material from a hydrocarbon plasma called quenched carbonaceous composite (QCC). A few years later, Papoular et al. (1989) was the first to use coals of different states of aromaticity to model the carriers of the aromatic IR bands (AIBs). Detailed studies of the spectral properties of HAC films have been continued by Duley (1994), Grishko \& Duley (2002), and Duley et al. (2005). In the last decade, the list of laboratory dust analogs was complemented by nanosized carbon grains produced by gas-phase condensation techniques such as laser pyrolysis (Herlin et al. 1998, Schnaiter et al. 1999, Jäger et al. 2006, Llamas et al. 2007, Jäger et al. 2007), resistive heating, arc discharge between carbon electrodes, or laser ablation coupled with condensation of grains in quenching gas atmospheres (Mennella et al. 1996, Schnaiter et al. 1998, Jäger et al. 1999, Jäger et al. 2008). 
New and interesting structures are onion-like carbon grains. Already some years ago, these grains have been proposed as the carrier of the interstellar UV bump (de Heer \& Ugarte 1993), however, the attribution remained questionable due to the shift of the measured UV absorption band in water. Onion-like carbon grains were also found in the granular and the dark QCC component produced by Wada et al. (1999). They found that the as-produced onions show a rather broad UV band around $220 \mathrm{~nm}$. Annealing experiments shifted the peak to longer wavelengths. Tomita et al. (2002) produced socalled defective carbon onions by annealing of nanodiamonds and found an absorption band at $3.9 \mu \mathrm{m}^{-1}$ for onions dispersed in water. Under annealing at temperatures higher than $1900 \mathrm{~K}$, polyhedral onion particles were formed, and a further absorption band appeared at $4.6 \mathrm{\mu m}^{-1}$. A theoretical model was developed by the authors to complement the experimental results. Defective onions of $5 \mathrm{~nm}$ with hollow cores of $0.7 \mathrm{~nm}$ in diameter were found to fit the interstellar UV bump very well. In contrast, Chhowalla et al. (2003) measured a narrow UV band at $4.55 \mu \mathrm{m}^{-1}$ for onion samples with sizes between 3 and $50 \mathrm{~nm}$ which were annealed at $873 \mathrm{~K}$ for $1 \mathrm{~h}$ in air. The results of the UV studies are rather controversial and further efforts are necessary to understand the UV absorption properties of onion-like carbon grains.

Kerogen, a material also found in primitive meteorites and interplanetary dust particles, has also been considered as a possible cosmic dust analog. On Earth, the name kerogen describes a family of polymer-like organic materials which are formed in sedimentation and annealing processes of hydrocarbons. Kerogen occurs in different states of aromaticity. Pendleton \& Allamandola (2002) have shown that the $3.4 \mu \mathrm{m}$ IR band of kerogen material extracted from the Murchison meteorite indicates a striking similarity to the $3.4 \mu \mathrm{m}$ IR profile of the diffuse interstellar medium (DISM), but there is no coincidence in the range between 5 and $10 \mu \mathrm{m}$. Papoular (2001) suggested that the $3.4 \mu \mathrm{m}$ as well as the accompanying MIR bands in protoplanetary and planetary nebulae and in the DISM could be carried by kerogen-like dust that is formed in circumstellar envelopes of evolved stars.

Generally, the different proposed dust analogs represent related carbonaceous materials composed of aromatic and saturated aliphatic structural units. Functional groups such as $-\mathrm{CH}_{x},=\mathrm{C}-\mathrm{H},-\mathrm{C}=\mathrm{O},-\mathrm{C}=\mathrm{C}-$, $\equiv \mathrm{C}-\mathrm{H},-\mathrm{C} \equiv \mathrm{C}-,-\mathrm{C}-\mathrm{O}-\mathrm{C}-$, and $-\mathrm{C}-\mathrm{OH}$, either incorporated into the structure of the structural units (SUs) or bound to the edges of these units, give rise to the appearance of observable IR bands. Therefore, in the laboratory, for analogs consisting of $\mathrm{C}$ and $\mathrm{H}$ and typical functional groups, similar IR bands can be observed. Differences in band positions result from differences in the chemical neighborhood of these functional groups which affect slightly the bond distances or electronic densities of the bonds. Varying band ratios are caused by varying abundances of these groups in the structure.

One of the key parameters for a complete understanding of the interstellar carbon dust component and its processing in the interstellar radiation field is the formation process of carbon grains. However, the formation pathway of carbonaceous matter in astrophysical environments, as well as in terrestrial gas-phase condensation reactions, is not yet understood. Knowledge of the detailed condensation conditions of carbon dust in astrophysical environments may help to predict the consequential formation pathways and the structural and morphological properties of the condensing carbonaceous material.

Carbonaceous materials form in large quantities in circumstellar envelopes around carbon stars. It is assumed that carbon soot and polycyclic aromatic hydrocarbons (PAHs) are simultaneously formed as nano- and subnanometer-sized particles via gas-phase condensation. Several authors (Frenklach \& Feigelson 1989, Cherchneff et al. 1992, Allain et al. 1997) have modeled the formation of PAHs in AGB stars. Whereas Frenklach \& 
Feigelson (1989) determined a small temperature range for the formation of PAHs in circumstellar environments, Cherchneff \& Cau (1999) reconsidered the modeling of the $\mathrm{PAH}$ and carbon dust formation in carbon-rich AGB stars based on a developed physicochemical model which describes the periodically shocked gas in the circumstellar shells close to the photosphere of the stars. The authors found that $\mathrm{C}_{6} \mathrm{H}_{6}$ formation begins at $1.4 \mathrm{R}_{*}$, and at a radius of $1.7 \mathrm{R}_{*}$ the conversion of single rings to PAHs at a temperature of around $1700 \mathrm{~K}$ starts. This temperature is much higher than the temperature window calculated in previous studies.

\section{Low- and high-temperature condensation of carbonaceous matter in the laboratory}

In our laboratory, we performed a series of gas-phase condensation experiments comprising laser ablation of graphite in quenching gas atmospheres and laser pyrolysis of hydrocarbons. We have performed our experiments in two different temperature regimes to achieve high-temperature (HT) and low-temperature (LT) condensation. The study of the formation pathways has been performed by analytical characterization of the condensates, including soot and its by-products. Laser ablation of graphite and subsequent condensation of carbonaceous matter has been carried out in quenching gas atmospheres of $\mathrm{He}$ or $\mathrm{He} / \mathrm{H}_{2}$ mixtures at low pressures. A more detailed description of the method can be found in Jäger et al. (2008). The second harmonic of a pulsed Nd:YAG laser was employed to evaporate carbon from a graphite target. The laser power densities varied between $2 \times 10^{8}$ and $9 \times 10^{9} \mathrm{~W} \mathrm{~cm}^{-2}$. The particles were extracted from the condensation zone by using a molecular beam technique.

Pulsed laser ablation of graphite in a quenching gas atmosphere is a complex nonequilibrium process, and sub-processes such as sublimation of the target material, ionization of the target species and the surrounding gas atoms and molecules, and deceleration of gas and condensates are rather difficult to describe. It is generally assumed that the temperature of the electrons, ions, and neutrals are different in an ablation plume. Therefore, we can only provide a lower limit of the temperature in the condensation zone. Our temperature estimation is based on different approaches. First we know that the sublimation of graphite at low pressures needs temperatures between 3600 and 4000 K. From the analysis of the HRTEM images of the condensates we can infer that no graphite was simply fractionalized or larger graphene layers ablated. Finally, the vibrational temperature of the laser-induced plasma generated by laser evaporation of a graphite target in a 10 mbar He atmosphere was found to range between 4000 and $6000 \mathrm{~K}$ for power densities between $0.5-2 \times 10^{9} \mathrm{~W} \mathrm{~cm}^{-2}$ (Iida \& Yeung 1994).

In addition, we have investigated different carbon condensates prepared by laserinduced pyrolysis (LIP) of gas-phase hydrocarbon precursors (ethylene, acetylene, and benzene) using either a pulsed or a continuous-wave (cw) $\mathrm{CO}_{2}$ laser. A detailed description of the experimental setup is given elsewhere (Llamas et al. 2007, Jäger et al. 2007). In the LIP experiments performed with the pulsed $\mathrm{CO}_{2}$ laser, $\mathrm{SF}_{6}$ was applied as a sensitizer. The laser radiation induces the dissociation of the reaction gas and the subsequent condensation of carbon nanoparticles which can either be extracted from the flow reactor by a particle beam extraction technique or by collection of the condensate in a filter. In the laser pyrolysis experiments with the cw laser, the temperatures in the condensation zone were determined with a pyrometer which provided a direct measurement of this important parameter. The temperatures achieved in pyrolysis experiments using pulsed $\mathrm{CO}_{2}$ lasers have already been investigated by several authors. At similar pyrolysis conditions, but slightly lower power densities, temperatures around $3000 \mathrm{~K}$ were 
Table 1. Experimental conditions for the HT and LT gas-phase condensation experiments.

\begin{tabular}{|c|c|c|c|c|c|c|}
\hline $\begin{array}{l}\text { Experi- } \\
\text { ment }\end{array}$ & Precursor & $\begin{array}{l}\text { Buffer } \\
\text { gas }\end{array}$ & Laser & $\begin{array}{c}\text { Laser power } \\
\text { density }\left(\mathrm{W} \mathrm{cm}^{-2}\right)\end{array}$ & $\begin{array}{l}\text { Tempera- } \\
\text { ture }(\mathrm{K})\end{array}$ & Condensate \\
\hline LA1 & Graphite & $\mathrm{He} / \mathrm{H}_{2}$ & pulsed & $2 \times 10^{8}-9 \times 10^{9}$ & $\geqslant 4000$ & $\begin{array}{l}\text { fullerene-like soot } \\
\text { and fullerenes }\end{array}$ \\
\hline LP1 & $\begin{array}{c}\mathrm{C}_{2} \mathrm{H}_{4}, \mathrm{C}_{2} \mathrm{H}_{2} \\
\mathrm{C}_{6} \mathrm{H}_{6}\end{array}$ & $\mathrm{He} / \mathrm{Ar}$ & pulsed & $1 \times 10^{7}-1 \times 10^{9}$ & $\geqslant 3500$ & $\begin{array}{l}\text { fullerene-like soot } \\
\text { and fullerenes }\end{array}$ \\
\hline LP2 & $\mathrm{C}_{2} \mathrm{H}_{4}, \mathrm{C}_{2} \mathrm{H}_{2}$ & $\mathrm{Ar}$ & $\mathrm{cw}$ & 5200 & & soot and $14 \mathrm{wt} \%$ PAHs \\
\hline LP3 & $\mathrm{C}_{2} \mathrm{H}_{4}, \mathrm{C}_{6} \mathrm{H}_{6}$ & Ar & $\mathrm{cw}$ & 5200 & $\sim 1500$ & soot and $33 \mathrm{wt} \%$ PAHs \\
\hline LP4 & $\mathrm{C}_{2} \mathrm{H}_{4}, \mathrm{C}_{6} \mathrm{H}_{6}$ & $\mathrm{Ar}$ & $\mathrm{cw}$ & 6400 & & soot and $17 \mathrm{wt} \% \mathrm{PAHs}$ \\
\hline LP5 & $\mathrm{C}_{2} \mathrm{H}_{4}$ & $\mathrm{Ar}$ & $\mathrm{cw}$ & 850 & $\sim 1000$ & $100 \mathrm{wt} \%$ PAHs \\
\hline
\end{tabular}
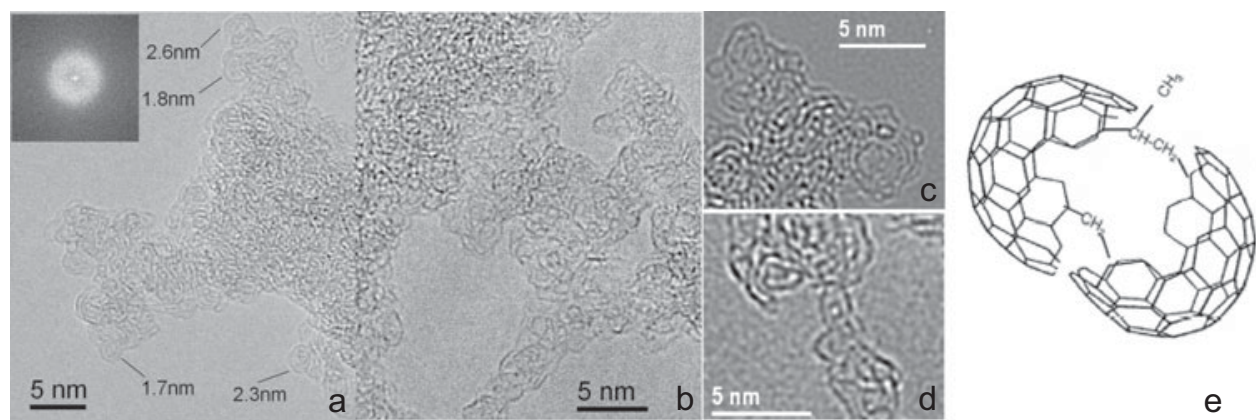

Figure 1. HRTEM images of fullerene-like carbon nano particles produced in a HT condensation process (LA1). Images a and b show soot grains with low and high hydrogen content, respectively. Images c and d present elongated fullerene particles and typical fullerene fragments. The structure e illustrates possible links between fullerene fragments in the soot condensates.

measured for comparable pressures of the He buffer gas (Kojima \& Naito 1981, Doubenskaia et al. 2006).

The preparation conditions for all gas-phase condensation experiments are compared in Table 1. The first two rows contain experiments performed with pulsed lasers characterized by high power densities and, consequently, high temperatures of more than $3500 \mathrm{~K}$ in the condensation zone. The condensation of particles in laser pyrolysis experiments employing a cw laser proceeds at much lower temperature (less than $1700 \mathrm{~K}$ ). We call these two different types of condensation HT and LT condensations, respectively.

\section{Soot formation and structural properties of the condensates}

HRTEM images of the carbonaceous matter produced in a HT gas-phase condensation process are shown in Figure 1.

The HRTEM micrographs reveal that very small fullerene-like particles are produced. These particles are composed of small, strongly bent graphene layers with varying lengths $\left(\mathrm{L}_{a}\right)$ and distances between these layers. The level of disorder depends on the employed condensation conditions which can be well observed in images a and b of Figure 1. Soot condensates produced only in helium atmospheres and at high pressures show more ordered and frequently closed fullerene cages. Grains containing higher contents of hydrogen are less ordered and do not show completely closed cages. In these grains, the cage fragments stick together by van der Waals forces or are linked by aliphatic $-\mathrm{CH}_{x}$ groups (see sketch e in Figure 1). The small size of the grains points to a strong supersaturation of 


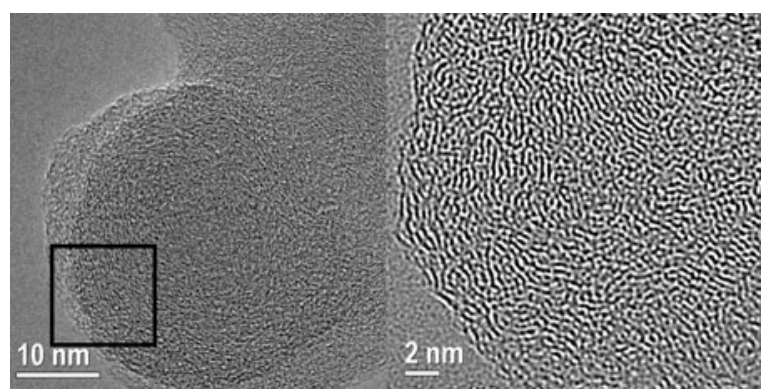

Figure 2. HRTEM images of typical large carbon nano particles produced in LT processes (LP2-4). The right micrograph displays a magnified section of the particle in the left image clearly showing the arrangement of the graphene layers in the particles.

carbon vapor in the condensation zone resulting in a high number of nucleation seeds. The further particle growth is exclusively due to coagulation.

A soot formation process, coupled with the formation of fullerenes, starting with polyyne chains, with subsequent spirocyclization and creation of saucer-shaped fullerene fragments has already been proposed by Kroto \& McKay (1988). The generation of fullerenes and fullerene snatches in the carbonaceous condensate could be verified by using electron microscopy. The critical point in the creation of bent graphene layers or disturbed fullerene-like structures is the formation of these cage fragments from the chains. Quantum chemical molecular dynamics simulations have shed some light on the formation of fullerenes from $\mathrm{C}_{2}$ fragments. High initial carbon densities are essential for such processes which can be found for example in carbon arc or laser ablation processes (Zheng et al. 2005). These authors found that the $\mathrm{C}_{2}$ molecules quickly combine to long and branched carbon chains and macromolecules for temperatures above $2000 \mathrm{~K}$. From the large chains, small cyclic structures with long carbon chains attached are formed (nucleation). The second step is a further ring condensation growth for example between two linear chains attached to a nucleus. Consequently, fullerene fragments of bowl shape with side chains are formed in this step (Irle et al. 2003).

In contrast, the condensed grains in LT processes are much larger and show more ordered and well developed planar graphene layers inside the particles (see Figure 2). Additionally, a mixture of PAHs was formed as by-products. This soluble component was analyzed by extraction of the soot in toluene, and the constituents of the extract were identified by chromatographic methods as a mixture of PAHs and partly hydrogenated PAHs. About 65 different PAHs have been analyzed, and 3-5 ring systems are by far most abundant. The PAHs are partly hydrogenated and contain saturated aliphatic- $\mathrm{CH}_{2}$ and $\mathrm{CH}_{3}$ groups. PAHs with masses up to 3000 Da were detected by application of matrix-assisted laser desorption/ionization mass spectrometry (MALDI TOF), but only in very low amounts. For example, a symmetric $\mathrm{PAH}$ molecule, $\mathrm{C}_{222} \mathrm{H}_{42}$, comprising 91 condensed rings and having a diameter of about $3 \mathrm{~nm}$ is a molecule of comparable mass. The large size and high internal order of the condensed soot grains points to a low supersaturation of the carbon vapor and the formation of a smaller number of stable nuclei compared to the HT condensation process. In the LT condensation, the further particle growth is dominated by condensation of intermediates on the surface of the seeds which means that PAHs continuously accumulate on the surface of the grains. Since larger PAHs have a lower volatility compared to the smaller ones, a preferred accumulation of large molecules during the surface growth process can be observed. The accumulation of the large molecules on the surfaces of the seeds can be confirmed by the results of the 

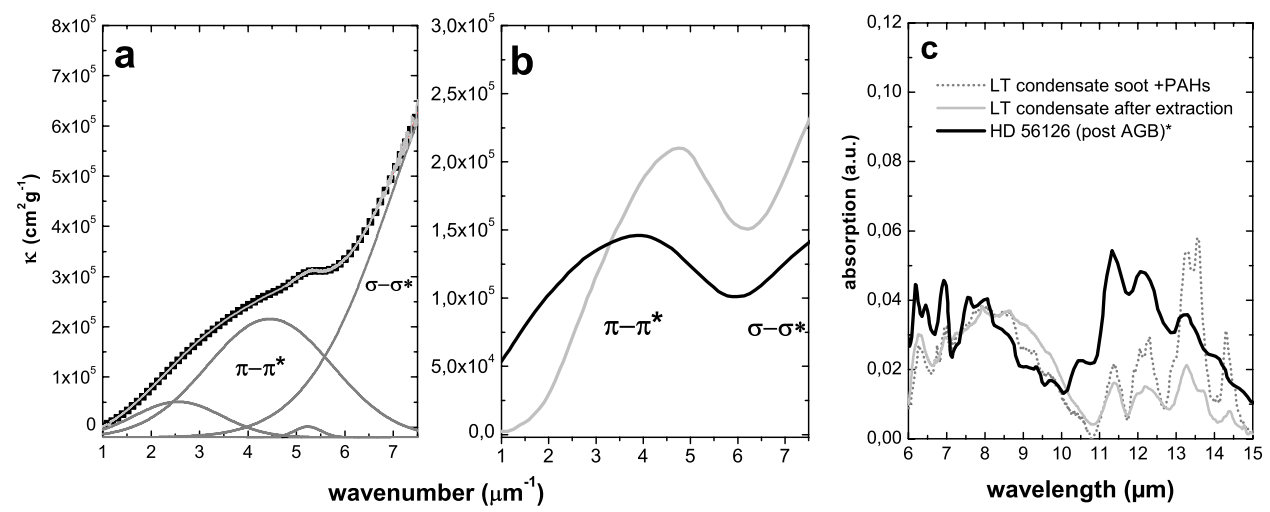

Figure 3. Comparison of the FUV/UV/VIS spectra of condensates produced in the HT (a) and LT (b) condensation process. A comparison between the IR spectral properties of a LT condensate with the observed spectrum of a post AGB star adapted from Hony et al. (2003) is shown in image (c).

HRTEM studies. A detailed analysis of the micrographs revealed a mean length $\mathrm{L}_{a}$ of the graphene sheets inside the particles of around $1.8 \mathrm{~nm}$ corresponding to PAHs with masses of around 1000 Da. The longest graphene layers have extensions of about $3 \mathrm{~nm}$ which corresponds to the highest-mass PAHs detected with MALDI TOF measurements.

\section{Spectral properties of the HT and LT condensates}

The spectral properties of the HT and LT condensates in the UV range differ considerably. Although the HT soot grains consist of about $50 \% s p^{2}$ hybridized carbon atoms the UV spectra do not show distinct UV absorption bands (see Figure 3a). A deconvolution of the UV/VIS absorption profile by employing four Gaussians reveals weak and broad bands due to $\pi-\pi^{*}$ transitions between 4.45 and $3.84 \mu \mathrm{m}^{-1}$, for the samples with the highest and the lowest hydrogen content, respectively. The weak Gaussian at approximately $2.5 \mu \mathrm{m}^{-1}$ is interpreted as a plasmon peak due to some larger, but strongly bent, graphene layers. The width of the $\left(\pi-\pi^{*}\right)$ main band and the appearance of the plasmon band accounts for a strong disorder in the carbon structures due to a broad distribution of curvatures and lengths of graphene layers in the small fullerene-like carbon grains (Llamas et al. 2007, Jäger et al. 2008). The very weak band at around $5.2 \mu \mathrm{m}^{-1}$ $(190 \mathrm{~nm})$ can be attributed to the absorption of $-\mathrm{C}=\mathrm{O}$ groups which could be identified in small amounts in the IR spectra of nearly all carbon soot samples. In contrast, the UV spectra of the LT condensates (Figure $3 \mathrm{~b}$ ) do show distinct UV absorption bands caused by $\left(\pi-\pi^{*}\right)$ transitions ranging between 3.6 and $4.9 \mu \mathrm{m}$. The exact position of this band depends on the internal structure of the soot grains and/or the content and composition of the soluble $\mathrm{PAH}$ component which also contributes to the spectra.

The IR spectral properties of the HT condensates are characterized by strong saturated and aliphatic $-\mathrm{CH}_{x}$ absorptions at $3.4 \mu \mathrm{m}$. No aromatic $=\mathrm{C}-\mathrm{H}$ has been observed in this spectral region. This supports the assumption that saturated aliphatic $-\mathrm{CH}_{x}$ groups are responsible for the links between the fullerene fragments. A weak feature at $5.8 \mu \mathrm{m}$ points to the incorporation of a small amount of $-\mathrm{C}=\mathrm{O}$ groups inside the carbon structure. The presence of aromatic $-\mathrm{C}=\mathrm{C}-$ groups can be identified at IR bands between 6.2 and $6.25 \mu \mathrm{m}$. Out-of-plane bending vibrations of aromatic $=\mathrm{C}-\mathrm{H}$ groups can be observed in the range between 11 and $14 \mu \mathrm{m}$ but their intensity is much lower compared to the LT condensates. Additionally, signatures for the presence of $-\mathrm{C} \equiv \mathrm{C}-$ triple bonds can be seen in the spectra at 3.03 and $4.7 \mu \mathrm{m}$ in in-situ measurements which points to the formation 
of polyynes as intermediates. A more detailed description of the IR spectral properties of soot particles produced in HT condensations can be found in Jäger et al. (2008).

As a consequence of the composition characteristic of the LT condensates which represent a mixture of soot and PAHs, aromatic IR bands (AIBs) such as 3.3, 6.2, 8.6, 11.3, 12.3 , and $13.3 \mu \mathrm{m}$ as well as aliphatic IR bands at $3.4,6.8$, and $7.25 \mu \mathrm{m}$ can be identified in the IR spectra of such condensates. As shown in Figure 3c, the AIBs and aliphatic IR bands are superimposed on two broad plateau features around 8 and $12 \mu \mathrm{m}$. The spectral characteristics of LT condensates strongly resemble the observed IR spectra of post AGB stars and protoplanetary nebulae (Kwok et al. 2001, Hony et al. 2003, Hrivnak et al. 2007). The comparison shown in Figure 3c clearly reveals that nearly all of the observed IR bands are also present in the LT condensate produced in the laboratory. Differences in band ratios, apparent for the $=\mathrm{C}-\mathrm{H}$ out-of-plane vibrational bands in the range between 11 and $14 \mu \mathrm{m}$ for the condensate, containing soot and PAHs, result from the presence of small PAHs in the condensed material. In particular, the bands at $13.3,13.5$, and $14.3 \mu \mathrm{m}$ are caused by $4-5$ adjacent $\mathrm{H}$ atoms bound to the aromatic ring and argue for smaller PAHs or for special PAHs containing phenyl rings bound to larger PAH molecules. The extracted soot particles which have consumed all the large PAHs during the growth process show a much better coincidence with the observed bands. The comparison reveals that the LT condensate is a promising dust analog for carbonaceous materials produced in carbon-rich AGB stars.

\section{Conclusions}

The results of our laboratory experiments have demonstrated that there are two different soot formation mechanisms. In HT condensations at temperatures higher than $3500 \mathrm{~K}$, very small fullerene-like particles and fullerenes, as by-products, are generated. The formation pathway of the soot is characterized by the formation of fullerene fragments from polyyne chains. LT condensates produced at temperatures lower than $1700 \mathrm{~K}$ consist of larger soot particles with long and rather plane graphene layers and mixtures of PAHs. Here, the formation process starts with a combination of small molecules resulting in the formation of aromatic benzene rings, the growth of larger and plane PAHs by subsequent $\mathrm{C}_{2}$ addition to the aromatic rings, and the final growth of grains by the condensation of large PAHs on the surfaces of the nuclei. Low-temperature condensation is a very likely formation process of soot and PAHs in AGB stars. Condensation temperatures in our laboratory studies were found to be very similar to the temperature range for carbon dust condensation in carbon-rich AGB stars predicted by Cherchneff \& Cau (1999).

\section{Acknowledgements}

This work has been supported by the Deutsche Forschungsgemeinschaft. We are grateful to Dr. I. Voicu for supplying some of the laser pyrolysis samples.

\section{References}

Allain, T., Sedlmayer, E., \& Leach, S. 1997, A\&A, 323, 163

Cherchneff, I., Barker, J. R., \& Tielens, A. G. G. M. 1992, ApJ, 413, 445

Cherchneff, I. \& Cau, P. 1999, in: T. Le Betre, A. Lèbre \& C. Waelkens (eds.), Asymptotic Giant Branch Stars, Proc. IAU Symposium No. 191 (San Francisco:ASP), p. 251

Chhowalla, M., Wang, H., Sano, N., Teo, K. B. K., Lee, S. B., \& Amaratunga, G. A. J. 2003, Phys. Rev. Lett., 90, 155504

de Heer, W. A. \& Ugarte, D. 1993, Chem. Phys. Lett., 207, 480

Doubenskaia, M., Bertrand, Ph., \& Smurov, I. 2006, Surface \& Coatings Technology, 201, 1955 
Duley, W. W. 1994, ApJ (Letters), 430, L133

Duley, W. W., Lazarev, S., \& Scott, A. 2005, ApJ, 620, L135

Duley, W. W. \& Williams, D. 1981, MNRAS, 196, 269

Frenklach, M. \& Feigelson, E. D. 1989, ApJ, 341, 372

Grishko, V. I. \& Duley, W. W. 2002, ApJ, 568, 448

Herlin, N., Bohn, I., Reynaud, C., Cauchetier, M., Galvez, A., \& Rouzaud, J.-N. 1998 AEA A 330, 1127

Hony, S., Tielens, A. G. G. M., Waters, L. B. F. M., \& de Koter, A. 2003, A\&\&A, 402, 211

Hrivnak, B. J., Geballe, T. R., \& Kwok, S. 2007, ApJ, 662, 1059

Iida, Y. \& Yeung, E. 1994, Appl. Spectr., 48, 945

Irle, S., Zheng, G., Elstner, M., \& Morokuma, K. 2003, Nano Letters 3, 1657

Jäger, C., Henning, Th., Schlögl, R., \& Spillecke, O. 1999, J. Non-Cryst. Solids 258, 161

Jäger, C., Huisken, F., Mutschke, H., Henning, Th., Poppitz, W., \& Voicu, I. 2007, Carbon, 45, 2981

Jäger, C., Krasnokutski, S., Staicu, A., Huisken, F., Mutschke, H., Henning, Th., Poppitz, W., \& Voicu, I. 2006, ApJS, 166, 557

Jäger, C., Mutschke, H., Henning, Th., \& Huisken, F. 2008, ApJ, submitted

Kojima, H. \& Naito, K. 1981, Ind. Eng. Chem. Prod. Res. Dev., 20, 396

Kroto, H. W. \& McKay, K. 1988, Nature, 331, 328

Kwok, S., Volk, K., \& Bernath, P. 2001, ApJ (Letters), 55, L87

Llamas-Jansa, I., Jäger, C., Mutschke, H., \& Henning, Th. 2007, Carbon, 45, 1542

Mennella, V., Colangeli, L., Palumbo, P., Rotundi, A., Schutte, W., \& Bussoletti, E. 1996, ApJ, 464, L191

Papoular, R. 2001, A\&A, 378, 597

Papoular, R., Conrad, J., Giuliano, M., Kister, J., \& Mille, G. 1989, A\&\&A, 217, 204

Pendleton, Y. J. \& Allamandola, L. J. 2002, ApJS, 138, 75

Sakata, A., Wada, S., Okutsu, Y., Shintani, H., \& Nakada, Y. 1983, Nature, 301, 493

Schnaiter, M., Henning, Th., Mutschke, H., Kohn, B., Ehbrecht, M., \& Huisken, F. 1999, ApJ, 519,687

Schnaiter, M., Mutschke, H., Dorschner, J., Henning, Th., \& Salama, F. 1998, ApJ, 498, 486

Tomita, S., Fujii, M., \& Hayashi, S. 2002, Phys. Rev. B, 66, 245424

Wada, S., Kaito, Ch., Kimura, S., Ono, H., \& Tokunaga, A. T. 1999 A\&A, 345, 259

Zheng, G., Irle, S., \& Morokuma, K. 2005, J. Chem. Phys., 122, 014708

\section{Discussion}

SAlama: Do you observe a continuous PAH size distribution, or do you instead observe many smaller PAHs.

JÄGER: PAHs with masses up to 3000 Da were detected in the MALDI-TOF mass spectra, but the smaller PAHs are by far the most abundant species.

SALAmA: Do you have any explanation for that?

JÄGER: My idea is that the large PAHs are consumed in the particle growth process. In low-temperature condensations, a small number of stable seed grains are formed simultaneously with a large amount of PAHs. The particle growth in the condensation under such conditions is dominated by a surface growth process which can be understood as a continuous accumulation of PAHs on the surfaces of the seed particles. The low-mass PAHs are much more volatile and they stay in the gas phase. The less volatile species accumulate on the surfaces of the nucleation seeds or small condensed particles and they built up the particles layer by layer. The largest graphene layers in the condensed particles were found to be around $3 \mathrm{~nm}$ corresponding to PAHs of approximately $3000 \mathrm{Da}$. I think this formation process explains why we do not see very abundant large PAHs. 